\title{
Nuevas estrategias farmacológicas para el tratamiento de cesación de tabaco
}

New pharmacological strategies for the treatment in smoking cessation

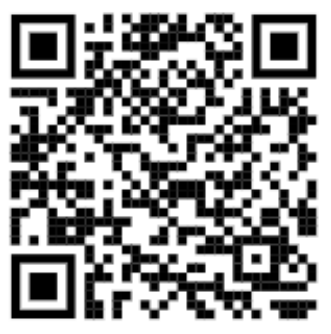

\author{
${ }^{1}$ Dr. Kevin Leiva Gabriel \\ Investigador independiente, San José Costa Rica \\ (D) https://orcid.org/0000-0003-4563-3525
}

${ }^{2}$ Dra. Valerie Quesada Vargas

Investigadora independiente, San José Costa Rica

(D) https://orcid.org/0000-0001-6653-621X

RECIBIDO

CORREGIDO

ACEPTADO

$08 / 04 / 2019$

$20 / 04 / 2019$

$04 / 05 / 2019$

\section{RESUMEN}

En la actualidad, el tabaquismo, continúa siendo la principal causa de enfermedades y muertes prevenibles a nivel mundial. El fumado al ser fisiológica y psicológicamente adictivo, persiste con tasas de éxito de abandono bajas. Como parte del proceso de cesado, la terapia farmacológica debe de ir estrictamente de la mano con la terapia conductual. La FDA ha aprobado tres fármacos de primera línea: la terapia de sustitución de nicotina (TSN), el Bupropión y la Vareniclina. Los inhaladores de nicotina han demostrado ser igual de eficaces que el resto de formas comerciales de TSN. El Bupropión, inicialmente comercializado como antidepresivo, mostró ser altamente efectivo en el cese del fumado, además de controlar la ganancia de peso durante el proceso. La Vareniclina es tratamiento de más alta eficacia, mostrando superioridad sobre la TSN y el Bupropión, debido a su efecto dual sobre receptores nicotínicos de acetilcolina.

PALABRAS CLAVE: reducción del hábito de fumar; formulaciones disuasorias del abuso; nicotina; bupropión; vareniclina.

\section{ABSTRACT}

${ }^{1}$ Médico general, graduado de la Universidad de Costa Rica (UCR). Código médico: 15918 Kevin.leivagabriel@ucr.ac.cr

${ }^{2}$ Médico general, graduada de la Universidad de Costa Rica (UCR). Código médico: 15888 Vdqv240@hotmail.com

Nowadays, smoking, remains the worldwide leading cause of death by preventable disease. As smoking is physiologically and psychologically addictive, the quitting success rates remain low. As part of the quitting process, the pharmacology therapy must go hand in hand with behavioral therapy. The FDA has approved three first-line drugs for smoking cessation: nicotine replacement therapy (NRT), bupropion and varenicline. Nicotine inhalers have been shown to be as effective as other commercial forms of nicotine replacement therapy. Bupropion, initially commercialize as an antidepressant, has shown to 
be highly effective in smoking cessation. This drug decreases the anxiety that patients experience when quitting smoking, in addition to control weight gain during the process. Varenicline is the treatment of higher efficacy, being superior than NRT and bupropion, due to its dual effect on nicotinic acetylcholine receptors.

KEYWORDS: smoking reduction; abuse - deterrent formulations; nicotine; bupropion; varenicline.

\section{INTRODUCCIÓN}

La importancia de la cesación de fumado mantiene su vigencia en la actualidad, ya que el fumado persiste como la principal causa de enfermedades y muertes prevenibles a nivel mundial (1), es el responsable de más de 6 millones de muertes anuales (2). Es considerado el factor de riesgo más importante en cuatro de las cinco principales causas de muerte: cardiopatía, cáncer, enfermedad pulmonar obstructiva crónica y enfermedades cerebrovasculares $(1,2,3,4)$.

El impacto del fumado sobreviene a que, a nivel mundial, del $10 \%$ al $30 \%$ de todas las muertes por enfermedad cardiovascular son atribuibles a fumar cigarrillos. Fumar tabaco afecta de manera adversa todas las fases del proceso de la enfermedad aterotrombótica, incluyendo disfunción endotelial, desarrollo y desestabilización de la placa ateroesclerótica, así como desequilibrio de factores antitrombóticos y protrombóticos que culminan en eventos cardiovasculares agudos (4).

Fumar es fisiológica y psicológicamente adictivo (1). El riesgo de adquirir dependencia en el hábito de fumar es similar al de la heroína y el consumo de alcohol (2). Esto se explica a que la nicotina, el componente psicoactivo principal del humo del tabaco, produce diversos efectos neurofisiológicos, motivacionales y de comportamiento a través de las interacciones con los receptores nicotínicos de acetilcolina en el sistema nervioso central. Estudios en gemelos y personas adoptadas, han sugerido que la adicción a la nicotina está estrechamente relacionada con factores ambientales y genéticos, aunque son estos últimos los que desempeñan un papel determinante en la propensión para el desarrollo de la adicción (5).

La principal problemática incide en que, aproximadamente el $70 \%$ de los fumadores activos, indican que les gustaría dejar de fumar, no obstante, del $40 \%$ de los fumadores que hacen un intento de dejar de fumar cada año, sólo alrededor del $5 \%$ experimenta éxito a largo plazo (3 a 12 meses). La mayoría de los consumidores de tabaco persisten en el consumo durante muchos años y típicamente ciclan a través de múltiples períodos de remisión y recaída, antes de que logren la abstinencia a largo plazo (3). Incluso la mayoría no deja de fumar hasta que han desarrollado complicaciones relacionadas con el tabaquismo (4).

Sin embargo, se ha demostrado que dejar de fumar es beneficioso para la salud a cualquier edad y aquellos 
fumadores de cigarrillos quienes renunciaron antes de los 35 años de edad tienen tasas de mortalidad similares a los que nunca fumaron (4).

Por lo tanto, los clínicos utilizan métodos para la cesación del fumado que incluyen: autoayuda, asesoramiento e intervenciones farmacológicas. La combinación de estos métodos (en especial asesoramiento e intervenciones farmacológicas) es más exitoso que la aplicación de cada uno por separado, aunque en la mayor parte de los casos son necesarios muchos intentos para obtener éxito (3).

Por todo lo antes mencionado, la finalidad de este artículo consiste en analizar los medicamentos de primera línea aprobados por Administración de Drogas y Alimentos de los Estados Unidos (FDA) para la cesación del fumado, como lo son la terapia de sustitución de nicotina (TSN), Bupropión y Vareniclina. El objetivo principal es sintetizar las nuevas investigaciones con respecto a mecanismos de acción, efectividad, riesgo cardiovascular, beneficios, efectos secundarios $y$ posología de estos fármacos, además de comparar estas características entre los mismos.

\section{MÉTODOS Y MATERIALES}

Para la elaboración de esta revisión, se utilizó bibliografía actualizadas y novedosas, de revistas con base científica, enfocándose en las últimas estrategias farmacológicas, probadas en estudios clínicos y aprobadas por la FDA para la cesación de fumado. Se tomó en cuenta el uso de bibliografía tanto en inglés como en español.

\section{INHALADORES DE NICOTINA}

La terapia de sustitución de nicotina (TSN) es una herramienta útil para la cesación del fumado, constituye uno de los medicamentos dentro de la línea farmacológica aprobados por la FDA, la cual debe además de complementarse de apoyo psicológico (4). La TSN se define como la administración de nicotina, por una vía alterna a la del consumo por cigarrillo, en una cantidad suficiente para disminuir los síntomas del síndrome de abstinencia, pero insuficientes para crear dependencia en el paciente (6).

Las formas comerciales de TSN son el chicle, parche transdérmico, spray nasal, inhaladores y tabletas. Todas han mostrado ser igualmente eficaces en ensayos clínicos (4). Según una revisión sistémica de Cochrane (7), se afirma que la TSN aumenta la tasa de cesación de fumado entre el $50 \%$ y $60 \%$ comparado con placebo.

Los inhaladores de nicotina están conformados por cartuchos que contienen $10 \mathrm{mg}$ de nicotina cada uno, que se coloca dentro de un cilindro de plástico hueco similar a un cigarro. Los cartuchos producen un vapor de nicotina que se calienta cuando el aire pasa a través de ellos. La absorción de la nicotina es en la mucosa oral, en lugar del sistema respiratorio (8). Cada cartucho contiene 80 puffs. La vía de administración es colocando el inhalador en la boca, no se debe inhalar hasta los pulmones. Se debe utilizar un cartucho cada 1 o 2 horas, máximo 16 al día. La terapia tiene una duración de aproximadamente 12 semanas (4).

Las versiones más recientes de los inhaladores producen un aumento en los 
niveles venosos de nicotina más rápido que la goma de mascar, con niveles de nicotina sanguíneos menores a un tercio de los niveles de los cigarros convencionales (8).

Son varios los beneficios relacionados con el inhalador de nicotina, el principal es que el usuario puede controlar la dosis de nicotina utilizada, además simula el movimiento de mano boca que se realiza al fumar cigarrillos, además que se puede adicionar al parche para solventar la ansiedad situacional (4).

No se han documentado efectos adversos serios, sin embargo, 50\% de los pacientes reportan irritación de boca y garganta, asimismo, de tos si se inhala muy profundamente. Además, las bebidas ácidas como el café disminuyen la absorción de la nicotina de la mucosa oral, por lo que se les recomienda a los usuarios utilizar el inhalador antes de consumir ese tipo de bebidas o evitarlas del todo (4).

\section{BUPROPIÓN}

Es un fármaco no nicotínico altamente eficaz para el tratamiento del tabaquismo, originalmente desarrollado y ampliamente utilizado como antidepresivo. Químicamente no está relacionado con los antidepresivos tricíclicos, los inhibidores selectivos de la recaptación de la serotonina ni otros antidepresivos conocidos (9).

Este es un medicamento de primera línea aprobado por la FDA para la cesación del fumado. Según un metaanálisis realizado por Cochrane (10) se ha demostrado aumentar el éxito del abandono significativamente hasta el final del tratamiento, con una OR de 1,71 a 1.99 versus el placebo (95\% IC 1.62.06). Otro estudio (9) que compara el uso de Bupropión de liberación sostenida versus placebo, demostró que a dosis diaria de $300 \mathrm{mg}, 44 \%$ de los pacientes desistieron del tabaco a las 7 semanas versus un $19 \%$ de los controles. $Y$ esta diferencia se mantuvo sostenidamente a lo largo de 12 meses.

Se sabe que actúa a nivel del núcleo accumbens inhibiendo la recaptación neuronal de dopamina; neurotransmisor implicado en el mecanismo de recompensa y gratificación. Este mecanismo explicaría la reducción de la ansiedad que experimentan los fumadores cuando lo utilizan (4). También inhibe en menor extensión la recaptación de norepinefrina en el núcleo cerúleos consiguiendo una reducción significativa de la intensidad de los síntomas del síndrome de abstinencia a la nicotina (11).

La eficacia del Bupropión se encuentra relacionada con la dosis, la concentración plasmática media y la concentración sanguínea de sus metabolitos (12). Los fumadores que utilizan Bupropión a dosis de 100mg, $150 \mathrm{mg}$ ó $300 \mathrm{mg}$ diarios tienen 1.42, 1.69 y 2.84 veces respectivamente más probabilidades de dejar de fumar que aquellos que reciben placebo.

Al cesar el fumado, gran parte de fumadores, principalmente las mujeres, tienen a aumentar de peso entre 4 y $5 \mathrm{~kg}$ en promedio (13) y en algunos casos aún más. Los mecanismos no se han dilucidado completamente, sin embargo, las investigaciones sugieren que es debido a la ausencia de nicotina, ya que esta actúa en los neurotransmisores del hipotálamo responsables de la regulación 
del apetito y la saciedad. Este puede ser un factor influyente en la decisión de la cesación de fumado, principalmente para las mujeres (9). En estos casos, el Bupropión puede ser una herramienta importante, ya que se ha demostrado que retrasa la ganancia de peso durante el tratamiento (10).

Otra indicación corresponde a pacientes con antecedentes de depresión que deseen dejar de fumar, ya que se ha demostrado ser eficaz en ambos aspectos (9).

Los pacientes deben comenzar el tratamiento cuando aún se está fumando, una o dos semanas antes del día de cesación (9). Se inicia con un comprimido de liberación prolongada de 150 mg por tres días, en la mañana. Luego, BID, uno en la mañana y otras 8 horas después y se continúa con esta dosis de 6 a 12 semanas. Si es necesario, el tratamiento se puede prolongar más tiempo. Algunos pacientes pueden perder las ganas de fumar o reducir espontáneamente su consumo antes de la fecha de abandono (12).

Al ser una droga estimulante del sistema nervioso central el efecto secundario más frecuente es el son insomnio (30-40\%), seguido por sequedad de boca (10\%) y náuseas (3-5\%), cefalea (3-17\%), manifestaciones alérgicas cutáneas (214\%) $(10,12)$.

El principal efecto adverso grave son las crisis convulsivas, estas se dan con una incidencia del $0.1 \%$ (5), por lo tanto, este medicamento está contraindicado en pacientes epilépticos o antecedentes de trastornos convulsivos. Asimismo, no se utiliza en pacientes con diagnóstico previo o actual de bulimia o anorexia nerviosa, ni pacientes con historia de uso inhibidores de la monoaminooxidasa en los 14 días previos (9).

El estudio CATS (Cardiovascular Safety of Varenicline, Bupropion, and Nicotine Patch in Smokers) (4) no encontró evidencia de efectos adversos negativos, ni aumento del riesgo cardiovascular en pacientes tratados con Bupropión versus placebo.

\section{VARENICLINA}

Dentro de las opciones farmacológicas aprobadas por la FDA, como estrategia de cesación de fumado, no nicotínicos, se encuentra la Vareniclina (Chantix®), siendo este el más efectivo en comparación con el Bupropión y la terapia de reemplazo de Nicotina $(14,15,16)$.

El mecanismo de acción se basa fundamentalmente en dos blancos terapéuticas; actuando como agonista parcial de receptores nicotínico de acetilcolina $\alpha 4 \beta 2$, en neuronas del sistema dopaminérgico mesolímbico (o centro de recompensa), áreas frontales y prelímbicas, incrementando los niveles de dopamina, así como actuando mediante una inhibición competitiva del receptor nicotínico, reduciendo los síntomas de abstinencia de la nicotina, por la liberación moderada de dopamina, y evitando el exceso en la liberación dopaminérgica ocasionados por la nicotina, al impedir la unión con su receptor $(17,18)$.

La Vareniclina, al igual que otras terapias sustitutivas, puede prescribirse en pacientes que aún no han consumido otras estrategias farmacológicas para el cese de fumado, o bien, que estas no han sido lo suficientemente eficientes, así como a pacientes que inician el 
tratamiento durante la disminución gradual del fumado que evidencien motivación para la suspensión del cigarrillo (18,19). A su vez, debe evitarse en pacientes en periodo de lactancia 0 embarazo, así como con aclaramientos endógenos de creatinina menores a $30 \mathrm{ml} / \mathrm{min}$, en los que se recomienda una reducción de dosis a 0,5mg por día (18).

El tratamiento con vareniclina se prescribe en un esquema de ajuste de dosis gradual, iniciando la primera semana con dosis de $0,5 \mathrm{mg}$ durante los primeros 3 días, y de $1 \mathrm{mg}$ de los días 4 al 7; posteriormente, debe establecerse el periodo de cese de fumado definitivo y continuar con dosis de $1,0 \mathrm{mg}$ dos veces al día, por dos a tres meses de forma continua, consumiéndose con alimentos para disminuir la presencia de náuseas como efecto adverso $(17,18)$.

Con respecto a la eficacia de la Vareniclina en el cese de fumado, en una revisión sistemática de 39 ensayos clínicos documentada por Cochrane, se probó la Vareniclina versus placebo, así como contra otras terapias sustitutivas (Bupropión y NRT), donde se determinó que a dosis estándar de Vareniclina, se incrementó la posibilidad de abandono del fumado cerca de dos a tres veces más, en contraste a la población que lo abandona sin asistencia farmacológica (20).

En la revisión de estudios clínicos, la vareniclina documentó un $R R$ de 2,24 y un OR de 2,88 para abstinencia a largo plazo (6 meses) con la dosis estándar de $1 \mathrm{mg}$ cada 12 horas, contra el placebo, así como un RR de 1,3 y OD de 1,59 contra la terapia sustitutiva con Bupropión y RR de 1,25 y OD de 1,57 contra la terapia de sustitución de nicotina, objetivando su efectividad en la suspensión del fumado $(16,17)$.

Dentro de los efectos adversos documentados con el uso de vareniclina, el más común es la presencia de náuseas (en cerca del 30-50\%) de los pacientes, así como trastornos del sueño, cefalea y efectos gastrointestinales, cuya incidencia varía con la dosis aplicada $(18,21)$.

Anteriormente, se creía la asociación de la Vareniclina con el riesgo de eventos cardiovasculares, así como neuropsiquiátricos, asociados con depresión o autolesiones (principalmente en pacientes con trastornos psiquiátricos de fondo), no obstante, en el ensayo clínico doble ciego, aleatorizado, controlado con placebo Eagle, se objetivan diferencias no significativas en el aumento de eventos neuropsiquiátricos atribuibles a la vareniclina, en contraste con el placebo $(21,22)$.

Por otra parte, con respecto a la asociación con eventos cardiovasculares, ensayos clínicos como SvanstrÖm y Prochaskra en 2012, demostrando la no asociación de vareniclina con mayor riesgo de isquemia miocárdica, enfermedad cerebrovascular, arritmias cardiacas, entre otros, con valores HR menores a 1, demostrando su eventual seguridad con respecto a riesgo cardiovascular $(17,22,23)$.

\section{CONCLUSIÓN}

Con la presente revisión bibliográfica, se concluye la relevancia de nuevas estrategias para lograr un cese en la práctica del fumado, la cual constituye un factor causal de gran morbimortalidad a 
nivel mundial. De forma general tanto los sustitutos nicotínicos con inhaladores, el Bupropion y la Vareniclina constituyen estrategias farmacológicas seguras para el cese del fumado, cuyos mecanismos de acción buscan fundamentalmente actuar en la disminución de síntomas asociado al síndrome de abstinencia mediante la administración controlada de Nicotina, en el caso de la terapia de sustitución de nicotina, así como actuando en vías dopaminérgicas relacionadas con las principales estructuras del sistema de recompensa neuronal. Las tres opciones demuestran alta eficacia como monoterapia por periodos de uso cercanos a las 12 semanas en dosis graduales, en comparación con placebo para la disminución del fumado, así como efectos secundarios mínimos y no asociación de riesgo cardiovascular en múltiples estudios clínicos, por lo que constituyen una vía segura y eficaz para el cese del fumado. Se debe recalcar que de forma comparativa, de las tres opciones, la Vareniclina constituye el medicamento con mayor eficacia, no obstante, se debe considerar la funcionalidad del Bupropion en pacientes que de forma concomitante poseen cuadros depresivos, o bien, donde este contribuya al control de peso posterior a la suspensión del fumado. Se recomienda recalcar la relevancia del uso conjunto de apoyo psicológico, así como crear consciencia en la importancia del compromiso por el cese de fumado para potenciar la adherencia a los esquemas de tratamiento.

\section{REFERENCIAS}

1. Vogeler T, McClain C, Evoy K. Combination bupropion SR and varenicline for smoking cessation: a systematic review. The American Journal of Drug and Alcohol Abuse. 2016; 42(2): 129139. https://doi.org/10.3109/00952990.2015.1117480

2. Benli A, Erturhan S, Oruc M, Kalpakci P, Sunay D, Demirel Y. A comparison of the efficacy of varenicline and bupropion and an evaluation of the effect of the medications in the context of the smoking cessation programme. Tobacco Induced Diseases. 2017; 15(February): 10.https://doi.org/10.1186/s12971-017$\underline{0116-0}$

3. Hakan T, Murat A, Özgür D, Metin Ç. Success Rate of Pharmacological Therapies Used for Smoking Cessation and Factors that Affect Smoking Cessation Rates. European Journal of General Medicine. 2015; 12(2): 125-130. https://doi.org/10.15197/sabad.1.12.26

4. Barua R, Rigotti N, Benowitz N, Cummings K, Jazayeri M, Morris P et al. 2018 ACC Expert Consensus Decision Pathway on Tobacco Cessation Treatment. Journal of the American College of Cardiology. 2018; 72(25): 3332-3365. https://doi.org/10.1016/j.jacc.2018.10.027

5. Liu M, Li X, Fan R, Liu X, Wang J. A Systematic Analysis of Candidate Genes Associated with Nicotine Addiction. BioMed Research International. 2015; 2015: 313709.https://doi.org/10.1155/2015/313709

6. Regalado-Pineda J, Lara-Rivas G, Osio-Echánove J, Ramírez-Venegas A. Tratamiento actual del tabaquismo. Salud Pública de México. 2007; 49(0036-3634): s270-s279.https://doi.org/10.1590/S0036$\underline{36342007000800018}$

7. Hartmann-Boyce J, Chepkin S, Ye W, Bullen C, Lancaster T. Nicotine replacement therapy versus control for smoking cessation. Cochrane Database of Systematic Reviews. 2018; (5): CD000146. https://doi.org/10.1002/14651858.CD000146.pub5

8. George T. Nicotine and Tobacco. Goldman-Cecil Medicine. 25th ed. Philadelphia: Elsevier/Saunders; 2016. p 145-149. 
9. Benowitz N, Brunetta P. Smoking Hazards and Cessation. Murray \& Nadel's textbook of respiratory medicine. 6th ed. Philadelphia: Elsevier; 2016. 807-821.e3.https://doi.org/10.1016/B978-1-4557-33835.00046-4

10. Cahill K, Stevens S, Perera R, Lancaster T. Pharmacological interventions for smoking cessation: an overview and network meta-analysis. Cochrane Database of Systematic Reviews. 2013; (5): CD009329. https://doi.org/10.1002/14651858.CD009329.pub2

11. Lerman C, Shields P, Wileyto E, Audrain J, Hawk L, Pinto A et al. Effects of dopamine transporter and receptor polymorphisms on smoking cessation in a bupropion clinical trial. Health Psychology. 2003; 22(5): 541-548. https://doi.org/10.1037/0278-6133.22.5.541

12. Díaz J, Jiménez C. Tratamiento farmacológico del tabaquismo. Información Terapéutica del Sistema Nacional de Salud. 2008; 32(3). p71-82.

13. Issa J, Santos $P$, Vieira L, Abe T, Kuperszmidt $C$, Nakasato $M$ et al. Smoking cessation and weight gain in patients with cardiovascular disease or risk factor. International Journal of Cardiology. 2014; 172(2): 485487. https://doi.org/10.1016/.i.jcard.2014.01.055

14. Bagdas D, Alkhlaif $Y$, Jackson A, Carroll F, Ditre J, Damaj M. New insights on the effects of varenicline on nicotine reward, withdrawal and hyperalgesia in mice. Neuropharmacology. 2018; 138: 7279. https://doi.org/10.1016/j.neuropharm.2018.05.025

15. Kazan T, Charntikov S. Individual differences in responding to bupropion or varenicline in a preclinical model of nicotine self-administration vary according to individual demand for nicotine. Neuropharmacology. 2019; 148: 139-150. https://doi.org/10.1016/j.neuropharm.2018.12.031

16. McDonough M. Update on medicines for smoking cessation. Australian Prescriber. 2015 ;38(4): 106111. https://doi.org/10.18773/austprescr.2015.038

17. Peña G. P, Zagolin B. M. Vareniclina. Revista chilena de enfermedades respiratorias. 2017; 33(3): 212215. https://doi.org/10.4067/s0717-73482017000300212

18. Hays J, Ebbert J. Varenicline for Tobacco Dependence. New England Journal of Medicine. 2008; 359(19): 2018-2024. https://doi.org/10.1056/NEJMct0800146

19. Steinberg M, Lu S, Williams J. Varenicline for smoking reduction in smokers not yet ready to quit: A double-blind, proof-of-concept randomized clinical trial. Addictive Behaviors. 2018; 84: 2026. https://doi.org/10.1016/j.addbeh.2018.03.026

20. Cahill K, Lindson-Hawley N, Thomas K, Fanshawe T, Lancaster T. Nicotine receptor partial agonists for smoking cessation. Cochrane Database of Systematic Reviews. 2016; (5): CD006103.https://doi.org/10.1002/14651858.CD006103.pub7

21. Anthenelli R, Benowitz N, West R, St Aubin L, McRae T, Lawrence D et al. Neuropsychiatric safety and efficacy of varenicline, bupropion, and nicotine patch in smokers with and without psychiatric disorders (EAGLES): a double-blind, randomised, placebo-controlled clinical trial. The Lancet. 2016; 387(10037): 2507-2520. https://doi.org/10.1016/S0140-6736(16)30272-0

22. Seguí Díaz M. ¿Cuáles son los efectos cardiovasculares y neuropsiquiátricos de la vareniclina en el tratamiento del tabaquismo?. SEMERGEN - Medicina de Familia. 2016; 42(6): e67e68. https://doi.org/10.1016/..semerg.2015.11.004

23. Kotz D, Simpson C, Viechtbauer W, van Schayck O, West R, Sheikh A. Cardiovascular and neuropsychiatric safety of varenicline and bupropion compared with nicotine replacement therapy for smoking cessation: study protocol of a retrospective cohort study using the QResearch general practice database. BMJ Open. 2014; 4(8): e005281-e005281. https://doi.org/10.1136/bmiopen-2014-005281 